\title{
Resistance to Echinocandins Complicates a Case of Candida albicans Bloodstream Infection: A Case Report
}

\author{
Laura Trovato ${ }^{1,2, * \mathbb{C}}$, Dafne Bongiorno ${ }^{2}$, Maddalena Calvo ${ }^{1,2}$, Giuseppe Migliorisi ${ }^{1,2}$, Albino Boraccino ${ }^{3}$, \\ Nicolò Musso ${ }^{2}$, , Salvatore Oliveri $^{2}$, Stefania Stefani ${ }^{2}{ }^{-1}$ and Guido Scalia ${ }^{1,2}$ \\ 1 U.O.C. Laboratory Analysis Unit, A.O.U. Policlinico-San Marco, 95123 Catania, Italy; \\ maddalenacalvo@gmail.com (M.C.); gpp.miglio@gmail.com (G.M.); lido@unict.it (G.S.) \\ 2 Department of Biomedical and Biotechnological Sciences, University of Catania, 95123 Catania, Italy; \\ dbongio@unict.it (D.B.); nmusso@unict.it (N.M.); oliveri@unict.it (S.O.); stefanis@unict.it (S.S.) \\ 3 U.O.C. Anesthesia and Intensive Care, Ospedale Garibaldi-Nesima, Azienda di Rilievo Nazionale e Alta \\ Specializzazione 'Garibaldi' Catania, 95122 Catania, Italy; albibor@gmail.com \\ * Correspondence: 1trovato@unict.it; Tel.: +39-0953781233
}

check for updates

Citation: Trovato, L.; Bongiorno, D.; Calvo, M.; Migliorisi, G.; Boraccino, A.; Musso, N.; Oliveri, S.; Stefani, S.;

Scalia, G. Resistance to Echinocandins Complicates a Case of Candida albicans Bloodstream Infection: A Case Report. J. Fungi 2021, 7, 405. https://doi.org/ 10.3390/jof7060405

Academic Editor: Sevtap

Arikan-Akdagli

Received: 31 March 2021

Accepted: 19 May 2021

Published: 21 May 2021

Publisher's Note: MDPI stays neutral with regard to jurisdictional claims in published maps and institutional affiliations.

Copyright: (c) 2021 by the authors. Licensee MDPI, Basel, Switzerland. This article is an open access article distributed under the terms and conditions of the Creative Commons Attribution (CC BY) license (https:/ / creativecommons.org/licenses/by/ $4.0 /)$.

\begin{abstract}
Invasive candidiasis is known to be one of the most common healthcare-associated complications and is caused by several Candida species. First-line drugs, particularly echinocandins, are effective, but there are increasing reports of resistance to these molecules, though rarely related to C. albicans. Even though the rate of echinocandins resistance remains low $(<3 \%)$, sporadic cases are emerging. Here, we present a case of bloodstream infection by a pan-echinocandin-resistant Candida albicans affecting a critically ill patient, who died in an intensive care unit following therapeutic failure and multiple organ dysfunction syndrome. This case highlights the need to suspect pan-echinocandin resistance in patients with prolonged echinocandin exposure, particularly in the presence of urinary tract colonization. Our study shows the importance of sequencing to predict therapeutic failure in patients treated with echinocandins and persistent candidemia.
\end{abstract}

Keywords: Candida albicans; bloodstream; echinocandins; resistance; mutations; FKS1

\section{Background}

Candida spp. normally colonizes healthy individuals asymptomatically, but it is also an opportunistic pathogen that can cause severe complications such as bloodstream infections [1]. The incidence of invasive candidiasis (IC) has increased significantly in recent years, with Candida albicans being the first cause of bloodstream infection followed by Candida glabrata. Patients on broad-spectrum antibiotics, immunosuppressed patients or patients with central venous access devices are more likely to develop fungal infections, including candidemia [2,3]. Antifungal therapy is a critical component of patient care, given the limited availability of antifungal drug classes. Echinocandins are recommended for the first-line treatment of invasive candidiasis, though in recent years Candida isolates with acquired resistance have been reported more frequently [3-7]. Echinocandins selectively inhibit glycosyltransferase $1,3-\beta$-D-glucan synthase, the enzyme responsible for the biosynthesis of a fundamental structural component of the cell wall, the oligosaccharide $1,3-\beta$-D-glucan. Today, three molecules are available with this function: caspofungin, micafungin and anidulafungin. Resistance to these molecules is predominantly associated with hotspot (HS) mutations in the 1,3- $\beta$-D-glucano synthase gene (FKS). Specifically for C. albicans, mutations responsible for echinocandins resistance were associated with two different hotspots in the FKS1 (or GSC1) portion [8]. However, with the exception of C. glabrata, resistance to echinocandins remains relatively low, being $<3 \%$ for Candida albicans and most Candida spp. $[9,10]$.

We report the case of a COVID 19-negative patient who developed bloodstream infection by a pan-echinocandin-resistant Candida albicans isolate. The patient died from 
multiple organ dysfunction syndrome before being able to benefit from a potentially effective antifungal therapy.

\section{Case Report and Results}

In May 2020, a 71-year-old man with a previous history of ischemic cardiac disease, stroke and hemiparesis was admitted to the Intensive Care Unit (ICU) of the Garibaldi Hospital in Catania for intensive monitoring after evacuation of a neck abscess. The patient was complaining of dysphagia and one-week fever treated with amoxicillin, corticosteroids and paracetamol. White blood cell count (WBC) was $28,700 / \mathrm{mm}^{3}, \mathrm{C}$-reactive protein (CRP) $20.40 \mathrm{mg} / \mathrm{dL}$, procalcitonin (PCT) $1.89 \mathrm{mcg} / \mathrm{L}$, creatinin $1.4 \mathrm{mg} / \mathrm{dL}$ and lactate $3.4 \mathrm{mmol} / \mathrm{L}$. Based on these findings, a septic event was suspected and empiric therapy with linezolid (1200 g/day) and piperacillin/tazobactam (18 g/day) was initiated. On the same day, a blood culture and bacteriological and mycological surveillance cultures (sputum and urinary samples, nasal and rectal swabs) were obtained. C. albicans was the only isolate detected in a nasal swab and in urinary and sputum samples. C. albicans was considered as a colonizer. A serum 1,3- $\beta$-D-glucan assay (Fungitell; Associates of Cape Cod Inc., Falmouth, MA, USA) was performed, revealing a positive result with a value of $114 \mathrm{pg} / \mathrm{mL}$. In consideration of the patient's medical history, a computed tomography (CT) of the neck and chest was performed on day 4 . Bronchoalveolar lavage (BAL) and new surveillance cultures (urinary sample, nasal and rectal swabs) were obtained to investigate the possible presence of an infectious disease causing pneumomediastinum. Candida albicans was isolated from a nasal swab as well as urinary and a BAL samples.

On day 7 , some inflammatory parameters were still high (WBC $24,800 / \mathrm{mm}^{3}$ and CRP $21.89 \mathrm{mg} / \mathrm{dL})$, but a decrease in PCT $(0.10 \mu \mathrm{g} / \mathrm{L})$ was also observed and the patient was no longer febrile. He was then moved to the otolaryngology unit with the indication to continue empiric therapy with linezolid and piperacillin/tazobactam and to start parenteral nutrition. On day 8 , due to worsening of general condition and a febrile episode, a new blood culture and several surveillance cultures (broncho-aspirate and urinary samples) were obtained. Parenteral nutrition and broad-spectrum treatment with linezolid (1200 mg/day) were continued, whereas piperacillin/tazobactam was switched to meropenem ( $3 \mathrm{~g} /$ day).

On day 9 , the patient developed respiratory failure and was moved to the thoracic surgery unit due to pleural effusion in the right lung. On day 13, positive blood cultures for Candida albicans were reported; the same was observed for broncho-aspirate and urine. According to the guidelines for the management of candidemia and on the basis of in vitro susceptibility to antifungal agents, clinicians decided to start caspofungin at $50 \mathrm{mg}$ daily (following a $70 \mathrm{mg}$ loading dose on day 1), even if no initial sensitivity test was performed to support this choice [11]. On day 28, the patient developed respiratory failure and the onset of multiple organ dysfunction syndrome and was moved back to the ICU (Figure 1). On day 29, BAL and new surveillance cultures (urinary sample, nasal and rectal swabs) were obtained and a Stenotrophomonas maltophilia strain was isolated from both the nasal swab and the BAL sample, while Candida albicans was isolated from the nasal swab and urinary sample). No COVID-19 genome was detected in the BAL sample. A new antibiotic therapy with linezolid (1200 $\mathrm{mg} /$ day), trimetroprim/sulfametoxazole (16 g/day) and fosfomycin (16 g/day) was initiated, while antifungal treatment with caspofungin was continued. On day 33 , serum levels of $\beta$-glucan were tested to monitor the course of the invasive infection, with a positive result of $>523 \mathrm{pg} / \mathrm{mL}$. On the same day, a new blood culture showed presence of Candida albicans (Isolate 2). On day 40, a new microbiological examination was performed on BAL, urinary and rectal samples: S. maltophilia was still isolated from the BAL sample, while the urinary sample revealed colonization by $C$. albicans and the rectal swab was positive for Acinetobacter baumannii. 


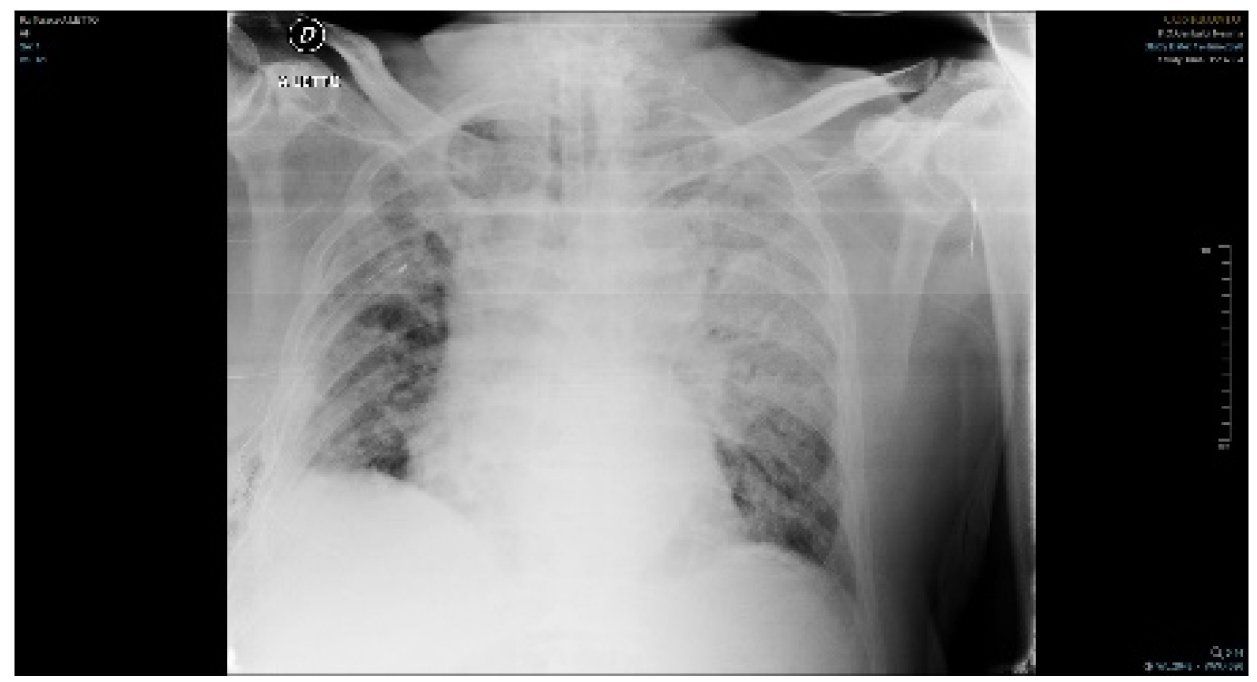

Figure 1. Chest radiograph shows several non-specific findings such as diffuse opacities, which correlate with clinical evidence of respiratory failure.

Antibiotic therapy with colistin (9 million IU/day) was added to the previous treatment. On day 42, a new blood culture showed presence of Candida albicans. On day 44, based on in vitro antifungal susceptibility results, caspofungin was replaced by amphotericin $B$ (400 mg/day). Unfortunately, the patient's clinical conditions worsened before he could possibly benefit from the antifungal therapy change. On day 46, the patient died of multiple organ dysfunction syndrome. The clinical/microbiological monitoring is summarized in Figure 2. Table 1 summarizes the results of antifungal susceptibility testing and FKS1 gene sequencing all both C. albicans isolated. Identification of the species was confirmed by Matrix-assisted laser desorption ionization time of flight mass spectrometry (MALDI-TOT MS) on a Microflex LT (Bruker Daltonics, Bremen, Germany) platform. Isolates 1 and 2 were uniformly susceptible, while Isolate 3 was resistant to anidulafungin, micafungin and caspofungin, with MICs of 1,4 and $8 \mu \mathrm{g} / \mathrm{mL}$, respectively (SensititreYeastOne ${ }^{\circledR}$ method; Thermo Fisher Scientific, Cleveland, OH, USA), according to the Clinical and Laboratory Standards (CLSI) clinical breakpoints [12]. Except for these three echinocandins, all isolates were susceptible to fluconazole (MIC, $0.25 \mathrm{mg} / \mathrm{L}$ ), itraconazole (MIC, $0.06 \mathrm{mg} / \mathrm{L}$ ), voriconazole (MIC, $<0.008 \mathrm{mg} / \mathrm{L}$ ), 5-fluorocytosine (MIC, $<0.06 \mathrm{mg} / \mathrm{L}$ ) and, according to the epidemiological cutoff values established by the CLSI [13], they also expressed wild-type susceptibility to amphotericin B (MIC, $0.5 \mathrm{mg} / \mathrm{L}$ ) and posaconazole (MIC, $0.03 \mathrm{mg} / \mathrm{L}$ ). The effectiveness of isavuconazole against all major Candida species has been evaluated [14]. According to these reports, the susceptibility test for isavuconazole was performed using the MIC strip method (Liofilchem, Roseto degli Abruzzi, TE, Italy) $[15,16]$ and showed an MIC of $0.5 \mathrm{mg} / \mathrm{L}$. However, the isolate could not be classified as either sensible or resistant to isavuconazole, according to the EUCAST breakpoint tables for the interpretation of MICs [17].

In consideration of the susceptibility results, molecular typing of the HS1 and HS2 of FKS1 genes was performed. For DNA extraction, the PathoNostics Extraction Kit was used following the manufacturer's instruction. DNA was quantified using the Qubit ${ }^{\circledR} 3.0$ Fluorometer (Cat No. Q33216, life Technolo-gies, Thermo Fisher Scientific, Monza, Italy) and the fluorimeter Qubit dsDNA BR Assay Kit (Cod. 32850, Invitrogen, Thermo Fisher Scientific, Monza, Italy). DNA was used for molecular typing of the HS1 and HS2 of FKS1 gene, performed using the Sanger method. The HS1 region was amplified using primers previously published [18]. Primers used in HS2 region amplification were: C.aFSK1HS2F 3' TGAGGATTGAAAATGAATGGGGA- ${ }^{\prime}$ and C.aFSK1HS2R $3^{\prime}$-GCTTTAGAAACACCACCT CTAGT- $5^{\prime}$; the amplicon size was $860 \mathrm{bp}, \mathrm{Tm} 60^{\circ} \mathrm{C}$. This set of primers was designed in house and analyzed with the online software: http:/ / www.premierbiosoft.com/netprimer/ (accessed on 27 July 2020). Both PCR amplifications were carried out in a Veriti Thermal Cycler (Applied Biosystems, Ther-moFisher, Monza, Italy) in a total volume of $25 \mu \mathrm{L}$ contain- 
ing $2 \times$ Multiplex PCR Master Mix (cat. No. BR0200804, biotechrabbit GmbH, Hennigsdorf, Germany), $10 \mathrm{ng}$ template DNA and verified agarose gel at $1.5 \%$ stained with SYBR Safe DNA Gel Stain (Cod. S33102, Invitrogen, Thermo Fisher Scientific, Monza, Italy). Obtained amplicons were purified, quantified and sequenced as previously described [19]. Obtained sequences were analyzed with BLAST (https:/ /blast.ncbi.nlm.nih.gov/Blast.cgi (accessed on 7 October 2020)) and UNIPROT (https:/ / www.uniprot.org/ (accessed on 7 October 2020)). Comparing our sequences to the reference strain (Ca22chr1A_C_albicans_SC 5314:505969.511662 (accessed on 7 October 2020)), we only found two homozygous singlenucleotide polymorphisms (SNP) in HS1, one of which is a silent mutation (A394G, reference strain position) that does not determine any alteration in the protein sequence. The other is a missense mutation (T398A, reference strain position) which was responsible for alteration in the 1,3- $\beta$-D-glucan protein sequence. In particular, the S645P mutation, as already described by Garnaud, results in transmembrane localization of the putative binding domain of the echinocandins, resulting in reduced affinity for these antifungals $[8,20]$.

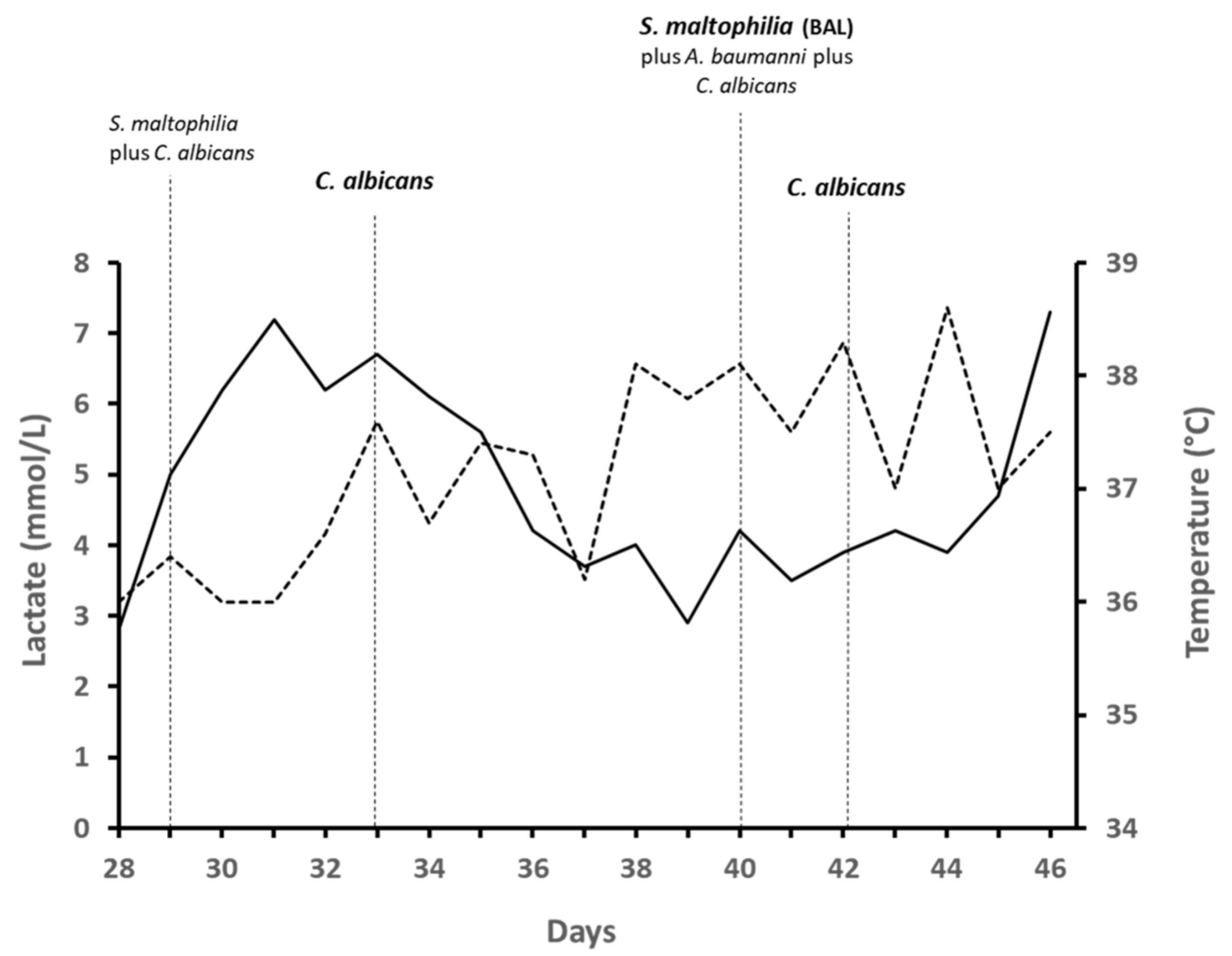

Figure 2. Clinical/microbiological monitoring during the patient's stay at the ICU. Temperature (dashed line) and lactate (solid line) trends are shown. Isolates from blood culture (bold) and surveillance cultures (not bold) are shown.

Table 1. Antifungal susceptibility testing and FKS1 gene sequencing for all C. albicans isolated.

\begin{tabular}{|c|c|c|c|c|c|c|c|c|c|c|c|c|c|}
\hline \multirow{2}{*}{ Isolate } & \multirow[t]{2}{*}{ Day } & \multicolumn{9}{|c|}{ MIC (mg/L) for Antifungal Agents } & \multicolumn{2}{|c|}{ MIC Strip (mg/L) } & \multirow{2}{*}{$\begin{array}{c}\begin{array}{c}\text { FKS Hotspot } \\
\text { Mutations }\end{array} \\
\text { FKS1 HS2 }\end{array}$} \\
\hline & & AMB & AND & CAS & MFG & FCT & ITC & VRC & POS & FLZ & ISA & FKS1 HS1 & \\
\hline C. albicans & 8 & 0.5 & 0.015 & 0.03 & 0.008 & $<0.06$ & 0.06 & $<0.008$ & 0.03 & 0.25 & 0.5 & Wild type & Wild type \\
\hline C. albicans & 33 & 0.5 & 0.015 & 0.03 & 0.008 & 0.008 & 0.06 & $<0.008$ & 0.03 & 0.25 & 0.5 & Wild type & Wild type \\
\hline C. albicans & 42 & 0.5 & 1 & 8 & 4 & 0.008 & 0.06 & $<0.008$ & 0.03 & 0.25 & 0.5 & S645P & - \\
\hline
\end{tabular}

MIC, minimum inhibitory concentration; AMB, amphotericin; AND, anidulafungin; CAS, caspofungin; MFG, micafungin; FCT, flucytosine; ITC, itraconazole; VRC, voriconazole, POS, Posaconazole; FLZ, fluconazole; ISA, isavuconazole. CLSI breakpoints [mg/L]: anidulafungin, caspofungin and micafungin $S \leq 0.25, R \geq 1$. 


\section{Discussion}

We reported the case of a patient who developed bloodstream infection by a panechinocandin-resistant Candida albicans isolate acquired following echinocandin exposure. Our patient had several risk factors for the development of invasive candidiasis (extensive use of antibiotics, protracted hospitalization, Candida colonization index $>0.5$ ). Candida albicans surface colonization of mucous membranes often represents the first stage of complication in a critical patient. The intensive care unit setting enables this yeast to invade the bloodstream, causing an increase in the mortality rate of hospitalized patients. Currently, the rate of echinocandin resistance remains low in this species and was mainly reported in isolates of Candida glabrata [21-24].

The main mechanism involved in echinocandin resistance of Candida spp. is related to mutations in hot-spot regions (HS1 and HS2) of the FKS1 gene, encoding the catalytic subunit of $\beta$-(1,3)-glucan synthase. The physiological role of the FKS2 and FKS3 genes in the onset of resistance among Candida species appears to be less important and is still widely debated [22]. Acquired mutations in the FKS1 and FKS2 genes have been predominantly found at position 645 (Serine), S645F (serine to phenylalanine), S645P (serine to proline), and S645Y (serine to tyrosine). Amino acid substitutions cause a change in the cell wall pattern, making it extremely difficult for echinocandins to hit their target. Mutations in the FKS genes are associated with high MIC values and low clinical response. These mutations usually result in pan-echinocandin resistance and affect mainly $C$. glabrata (prevalence range $2-13 \%)$ and, more rarely, C. albicans $(<1 \%)[6,25]$. In our case, a missense mutation S645P was observed in the HS1 region of the FKS1 gene of the Candida albicans isolate (Isolate 3), associated to echinocandin resistance. Echinocandin resistance can rapidly develop after initiation of treatment, but it can also appear after prolonged therapy, and caspofungin is associated with a higher risk of inducing FKS mutations in comparison to other echinocandins $[23,26]$. According to the literature data, we can say that echinocandin resistance is indeed more common in patients with long hospitalization and recurrent candidemia undergoing prolonged antifungal treatment $[25,27]$. In our case, the patient was put on caspofungin on day 13 , as the first isolate of $C$. albicans susceptible to all antifungal agents tested was detected from blood cultures (Isolate 1). Following worsening of clinical conditions, respiratory failure and multiple organ dysfunction, the patient was moved back to the ICU and continued on caspofungin. On day 33, a new episode of candidemia was discovered: C. albicans was still detected (Isolate 2) and its susceptibility pattern was the same as in the first one. Only on day 42 did our patient develop an episode of candidemia with an echinocandin-resistant isolate of $C$. albicans (Isolate 3), following prolonged therapy with caspofungin (29 days). We demonstrated that Isolate 3 , compared to Isolates 1 and 2 (wild type), had increased MICs for echinocandins, and all values were higher than the CLSI resistance breakpoints [12]. Besides prolonged antifungal treatment with echinocandins, another risk factor for the development of acquired FKS mutations is the potential existence of hidden reservoirs of Candida spp. (on abdominal cavity and mucosal surfaces), where there is no regular drug penetration [28,29]. Probably, in our case, the persistent urinary tract colonization also represented a risk factor for acquired FKS mutations and the appearance of echinocandins resistance during treatment further aggravated the adverse prognosis of candidemia. Despite early replacement of antifungal treatment, the patient died in two days due to the general worsening of his clinical conditions. This case further confirms the importance of quickly performing susceptibility tests upon isolation of Candida spp from sterile sites, especially when the infection appears not to respond to the antifungal treatment for a long time, as also stated in the current guidelines for the management of candidemia and invasive candidiasis of the Infectious Diseases Society of America (IDSA) [11]. Due to significant interlaboratory variability for caspofungin MICs, routine testing derived by CLSI or EUCAST methodology may lead to incorrect results especially when the isolate was detected to be resistant. Accordingly, molecular analysis is recommended for confirmation of resistance. Furthermore, SensititreYeastOne ${ }^{\circledR}$ method should be routinely used to test in vitro susceptibility to caspofungin due to low MIC variability [30]. 
In conclusion, this case highlights that even though antifungal resistance in C. albicans is uncommon, individual isolates may not necessarily follow this general pattern and that susceptibility testing, as well as FKS sequencing, are indispensable to predict therapeutic failure in patients treated with echinocandins and persistent candidemia.

Author Contributions: Conceptualization, L.T. and M.C.; methodology, D.B., N.M., L.T., M.C. and G.M.; investigation, D.B., N.M., L.T., M.C. and G.M.; data curation, L.T., M.C., A.B., S.S.; writingoriginal draft preparation, L.T., M.C., G.M., D.B. and S.S.; writing-review and editing, S.S., S.O. and G.S.; supervision, L.T. and S.S. All authors have read and agreed to the published version of the manuscript.

Funding: This research received no external funding.

Institutional Review Board Statement: Not applicable.

Informed Consent Statement: Not applicable.

Data Availability Statement: All data generated or analyzed during this study are included in this published article.

Conflicts of Interest: The authors declare no conflict of interest.

\section{References}

1. Lohse, M.B.; Gulati, M.; Johnson, A.D.; Nobile, C.J. Development and regulation of single- and multi-species Candida albicans biofilms. Nat. Rev. Microbiol. 2018, 16, 19-31. [CrossRef] [PubMed]

2. Pfaller, M.A.; Diekema, D.J.; Turnidge, J.D.; Castanheira, M.; Jones, R.N. Twenty years of the SENTRY antifungal surveillance program: Results for Candida species from 1997-2016. Open Forum Infect. Dis. 2019, 6, S79-S94. [CrossRef]

3. Arastehfar, A.; Yazdanpanah, S.; Bakhtiari, M.; Fang, W.; Pan, W.; Mahmoudi, S.; Pakshir, K.; Daneshnia, F.; Boekhout, T.; Ilkit, M.; et al. Epidemiology of candidemia in Shiraz, southern Iran: A prospective multicenter study (2016-2018). Med. Mycol. 2021, 59, 422-430. [CrossRef] [PubMed]

4. Cornely, O.A.; Bassetti, M.; Calandra, T.; Garbino, J.; Kullberg, B.J.; Lortholary, O.; Meersseman, W.; Akova, M.; Arendrup, M.C.; Arikan-Akdagli, S.; et al. ESCMID* guideline for the diagnosis and management of Candida diseases 2012: Non-neutropenic adult patients. Clin. Microbiol. Infect. 2012, 18, 19-37. [CrossRef] [PubMed]

5. Ullmann, A.J.; Akova, M.; Herbrecht, R.; Viscoli, C.; Arendrup, M.C.; Arikan-Akdagli, S.; Bassetti, M.; Bille, J.; Calandra, T.; Castagnola, E.; et al. ESCMID* guideline for the diagnosis and management of Candida diseases 2012: Adults with haematological malignancies and after haematopoietic stem cell transplantation (HCT). Clin. Microbiol. Infect. 2012, 18, 53-67. [CrossRef]

6. Alexander, B.D.; Johnson, M.D.; Pfeiffer, C.D.; Jimenez-Ortigosa, C.; Catania, J.; Booker, R.; Castanheira, M.; Messer, S.A.; Perlin, D.S.; Pfaller, M.A. Increasing echinocandin resistance in Candida glabrata: Clinical failure correlates with presence of FKS mutations and elevated minimum inhibitory concentrations. Clin. Infect. Dis. 2013, 56, 1724-1732. [CrossRef]

7. Pfaller, M.A.; Messer, S.A.; Hollis, R.J.; Boyken, L.; Tendolkar, S.; Kroeger, J.; Diekema, D.J. Variation in susceptibility of bloodstream isolates of Candida glabrata to fluconazole according to patient age and geographic location in the United States in 2001 to 2007. J. Clin. Microbiol. 2009, 47, 3185-3190. [CrossRef]

8. Spettel, K.; Barousch, W.; Makristathis, A.; Zeller, I.; Nehr, M.; Selitsch, B.; Lackner, M.; Rath, P.M.; Steinmann, J.; Willinger, B. Analysis of antifungal resistance genes in Candida albicans and Candida glabrata using next generation sequencing. PLoS ONE 2019, 14, e0210397. [CrossRef]

9. Perlin, D.S. Echinocandin resistance in candida. Clin. Infect. Dis. 2015, 61, S612-S617. [CrossRef]

10. Castanheira, M.; Woosley, L.N.; Diekema, D.J.; Messer, S.A.; Jones, R.N.; Pfaller, M.A. Low prevalence of fks1 hot spot 1 mutations in a worldwide collection of Candida strains. Antimicrob. Agents Chemother. 2010, 54, 2655-2659. [CrossRef]

11. Pappas, P.G.; Kauffman, C.A.; Andes, D.R.; Clancy, C.J.; Marr, K.A.; Ostrosky-Zeichner, L.; Reboli, A.C.; Schuster, M.G.; Vazquez, J.A.; Walsh, T.J.; et al. Clinical practice guideline for the management of candidiasis: 2016 Update by the infectious diseases society of America. Clin. Infect. Dis. 2016, 62, e1-e50. [CrossRef]

12. Clinical and Laboratory Standards Institute. Reference Method for Broth Dilution Antifungal Susceptibility Testing of Yeasts; Approved Standard CLSI Document M27-A3; Clinical and Laboratory Standards Institute: Wayne, PA, USA, 2008.

13. Clinical and Laboratory Standards Institute. Epidemiological Cutoff Values for Antifungal Susceptibility Testing; CLSI Supplement M59; Clinical and Laboratory Standards Institute: Wayne, PA, USA, 2018.

14. Ellsworth, M.; Ostrosky-Zeichner, L. Isavuconazole: Mechanism of action, clinical efficacy, and resistance. J. Fungi 2020, $29,324$.

15. Howard, S.J;; Lass-Flörl, C.; Cuenca-Estrella, M.; Gomez-Lopez, A.; Arendrup, M.C. Determination of isavuconazole susceptibility of Aspergillus and Candida species by the EUCAST method. Antimicrob. Agents Chemother. 2013, 57, 5426-5431. [CrossRef]

16. Trovato, L.; Scalia, G.; Palermo, C.I.; Costanzo, C.M.; Oliveri, S. Evaluation of isavuconazole MIC strips for susceptibility testing of Aspergillus and Scedosporium species. Med. Mycol. 2019, 1, 429-433. [CrossRef] 
17. European Committee on Antimicrobial Susceptibility Testing. Breakpoint Tables for Interpretation of Mics for Antifungal Agents. Version 10.0. Available online: https:/ / www.eucast.org/astoffungi/clinicalbreakpointsforantifungals / (accessed on 4 February 2020).

18. Kritikos, A.; Neofytos, D.; Khanna, N.; Schreiber, P.W.; Boggian, K.; Bille, J.; Schrenzel, J.; Mühlethaler, K.; Zbinden, R.; Bruderer, T.; et al. Accuracy of sensititre yeast one echinocandins epidemiological cut-off values for identification of fks mutant candida albicans and candida glabrata: A ten year national survey of the fungal infection network of Switzerland (FUNGINOS). Clin. Microbiol. Infect. 2018, 24, 1214.e1. [CrossRef]

19. Musso, N.; Costantino, A.; La Spina, S.; Finocchiaro, A.; Andronico, F.; Stracquadanio, S.; Liotta, L.; Visalli, R.; Emmanuele, G. mNew SARS-CoV-2 Infection detected in an Italian pet cat by RT-qPCR from deep pharyngeal swab. Pathogens 2020, 11, 746.

20. Gagliano, M.; Marchiani, C.; Bandini, G.; Bernardi, P.; Palagano, N.; Cioni, E.; Finocchi, M.; Bellando, S.; Moggi, A. A rare case of Candida glabrata spondylodiscitis: Case report and literature review. Int. J. Infect. Dis. 2018, 68, 31-35. [CrossRef]

21. Costa-de-Oliveira, S.; Rodrigues, A.G. Candida albicans antifungal resistance and tolerance in bloodstream infections: The triad yeast-host-antifungal. Microorganisms 2020, 22, 154. [CrossRef]

22. Suwunnakorn, S.; Wakabayashi, H.; Kordalewska, M.; Perlin, D.S.; Rustchenko, E. FKS2 and FKS3 genes of opportunistic human pathogen candida albicans influence echinocandin susceptibility. Antimicrob. Agents Chemother. 2018, 62, e02299. [CrossRef]

23. Perlin, D.S. Echinocandin resistance, susceptibility testing and prophylaxis: Implications for patient management. Drugs 2014, 14, 1573-1585. [CrossRef]

24. Posteraro, B.; Torelli, R.; Vella, A.; Leone, P.M.; De Angelis, G.; De Carolis, E.; Ventura, G.; Sanguinetti, M.; Fantoni, M. Panechinocandin-resistant Candida glabrata bloodstream infection complicating COVID-19: A fatal case report. J. Fungi 2020, 6, 163. [CrossRef] [PubMed]

25. Shields, R.K.; Nguyen, M.H.; Press, E.G.; Cumbie, R.; Driscoll, E.; Pasculle, A.W.; Cornelius, J. Rate of FKS mutations among consecutive candida isolates causing bloodstream infection. Antimicrob. Agents Chemother. 2015, 59, 7465-7470. [CrossRef] [PubMed]

26. Shields, R.K.; Kline, E.G.; Healey, K.R.; Kordalewska, M.; Perlin, D.S.; Nguyen, M.H.; Cornelius, J. Spontaneous Mutational Frequency and FKS Mutation Rates Vary by Echinocandin Agent against Candida glabrata. Antimicrob. Agents Chemother. 2019, 21, e01692. [CrossRef] [PubMed]

27. Beyda, N.D.; John, J.; Kilic, A.; Alam, M.J.; Lasco, T.M.; Garey, K.W. FKS mutant Candida glabrata: Risk factors and outcomes in patients with candidemia. Clin. Infect. Dis. 2014, 59, 819-825. [CrossRef]

28. Shields, R.K.; Nguyen, M.H.; Press, E.G.; Clancy, C.J. Abdominal candidiasis is a hidden reservoir of echinocandin resistance. Antimicrob. Agents Chemother. 2014, 58, 7601-7605. [CrossRef]

29. Healey, K.R.; Nagasaki, Y.; Zimmerman, M.; Kordalewska, M.; Park, S.; Zhao, Y.; Perlin, D.S. The gastrointestinal tract is a major source of echinocandin drug resistance in a murine model of Candida glabrata colonization and systemic dissemination. Antimicrob. Agents Chemother. 2017, 61, e01412-e01417. [CrossRef]

30. Espinel-Ingroff, A.; Alvarez-Fernandez, M.; Cantón, E.; Carver, P.L.; Chen, S.C.; Eschenauer, G.; Getsinger, D.L.; Gonzalez, G.M.; Govender, N.P.; Grancini, A.; et al. Multicenter study of epidemiological cutoff values and detection of resistance in Candida spp. to anidulafungin, caspofungin, and micafungin using the Sensititre YeastOne colorimetric method. Antimicrob. Agents Chemother. 2015, 59, 6725-6732. [CrossRef] 